\title{
Doppler-free two-photon absorption of two coherent beams
}

\author{
Ming Chiang Li \\ Department of Physics, Virginia Polytechnic Institute and State University, Blacksburg, Virginia 24061 \\ (Received 8 July 1977)
}

\begin{abstract}
Proposed and performed experiments on the Doppler-free two-photon absorption of two coherent laser beams are discussed. The coherent phenomenon in experiments follows directly from basic quantum mechanics. It is shown that, to excite the coherent absorption, the laser beams need not be spatially or temporally separated.
\end{abstract}

Recently, there has been considerable interest in the coherent Doppler-free two-photon absorption process. $^{1}$ The main characteristic of this process is that after absorbing two opposite-momentum photons the atom's momentum remains unchanged. In other words, the atomic motion will not inhibit the coherence of the absorption. Hence, a new resonance phenomenon occurs. It is a purely quantum-mechanical phenomenon and does not depend on the detailed dynamics. ${ }^{2}$ Several experiments have been suggested to observe this resonance phenomenon. In that proposed by Baklanov, Dubetskii, and Chebotayev, ${ }^{3}$ a beam of atoms interacts with two spatially separated laser beams. The width of the predicted resonance depends on the time of flight of an atom between the two laser beams. The resonance fringe variation can be expressed as

$$
\cos \left[\left(\omega_{0}-2 \omega\right) T\right]
$$

where $T$ is the time of flight, $\omega_{0}$ the Bohr frequency of the atomic transition, and $\omega$ the laser frequency. This resonance is similar to the radio-frequency Ramsey resonance and these fringes, at present, are referred to as Ramsey fringes. ${ }^{4}$

In another proposed experiment, ${ }^{2}$ atomic and laser beams intersect in a common spatial region. The experimental arrangement is shown in Fig. 1. $\mathrm{SM}$ is the laser-beam splitter. $\mathrm{M}_{1}, \mathrm{M}_{1}^{\prime}, \mathrm{M}_{2}$, and $\mathrm{M}_{2}^{\prime}$ are the reflecting mirrors. The atomic beam passes through the intersection of the laser beams and is perpendicular to the paper. The Dopplerfree transition amplitude in this experiment is proportional to

$$
\begin{aligned}
& g\left(\omega_{0}\right)\left(\left\langle\overrightarrow{\mathrm{p}}_{1}, \epsilon_{2}|t| \overrightarrow{\mathrm{p}}_{1}, \epsilon_{1} ; \overrightarrow{\mathrm{k}}_{1} ;-\overrightarrow{\mathrm{k}}_{1}\right\rangle\right. \\
& \left.\quad+a e^{2 i k L}\left\langle\overrightarrow{\mathrm{p}}_{1}, \epsilon_{2}|t| \overrightarrow{\mathrm{p}}_{1}, \epsilon_{1} ; \overrightarrow{\mathrm{k}}_{2} ;-\overrightarrow{\mathrm{k}}_{2}\right\rangle\right),
\end{aligned}
$$

where $g\left(\omega_{0}\right)$ describes the atomic line profile. ${ }^{5}$ $\left\langle\overrightarrow{\mathrm{p}}_{1}, \epsilon_{2}|t| \overrightarrow{\mathrm{p}}_{1}, \epsilon_{1} ; \overrightarrow{\mathrm{k}}_{i} ;-\overrightarrow{\mathrm{k}}_{\boldsymbol{i}}\right\rangle$ for $i=1,2$ is the reduced transition amplitude. In the transition, the momentum $\vec{p}_{1}$ of the atom remains the same while its internal energy is changed from $\epsilon_{1}$ to $\epsilon_{2}$,

$$
\epsilon_{2}-\epsilon_{1}=\hbar \omega_{0}
$$

The wave vectors of the laser beams are indicated in Fig. 1 with

$$
\left|\overrightarrow{\mathrm{k}}_{1}\right|=\left|\overrightarrow{\mathrm{k}}_{2}\right|=k=\omega / c,
$$

where $c$ is the velocity of light. The real quantity $a$ in Eq. (2) is the relative strength of the two laser beams after the beam splitter. $L$ is the path length difference between path $S M-M_{1}-M_{1}^{\prime}$ and path $S M$ $-M_{2}-M_{2}^{\prime}$. The predicted resonance fringe variation in this experiment can be expressed $\mathrm{as}^{2}$

$$
\cos (2 k L+\Phi) \text {, }
$$

where $\Phi$ is the relative phase between the reduced transition amplitudes in Eq. (2). Due to the dependence on the relative phase $\Phi$, this experiment provides a way to measure the phase of the reduced transition amplitude directly. ${ }^{6}$

Recently a very interesting experiment ${ }^{7}$ was performed with a pulsed laser beam which was interpreted as a variation of the separated beam experiment proposed by Baklanov, Dubetskii, and Chebotayev. I want to emphasize here that the experiment is in fact intrinsically related to the crossedbeam experiment mentioned above. In the experiment performed, two laser beams were overlaid with

$$
\overrightarrow{\mathrm{k}}_{1}=\overrightarrow{\mathrm{k}}_{2}=\overrightarrow{\mathrm{k}}
$$

and a pulsed laser was used. By overlaying the laser beams, the alignment difficulties present in the

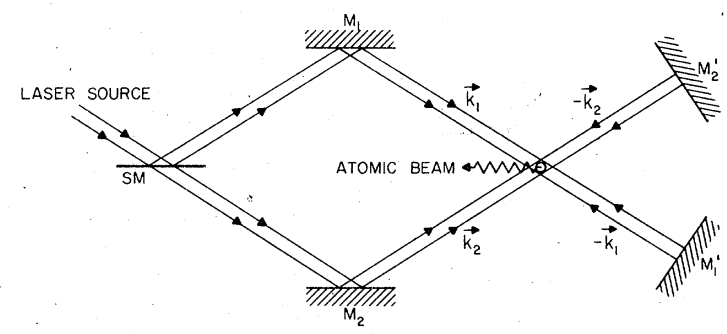

FIG. 1. SM is the laser-beam splitter, $M_{1}, M_{1}^{\prime}, M_{2}$, and $\mathrm{M}_{2}^{\prime}$ are the reflecting mirrors. The atomic beam is perpendicular to the paper and passes through the intersection of the laser beams. 
crossed-beam experiment are reduced. By using a pulsed laser, beam intensities are increased.

The Doppler-free transition amplitude in the experiment performed is

$\int d k^{\prime} G^{2}\left(k^{\prime}\right) g\left(\omega_{0}\right)\left(1+a e^{2 i k^{\prime} L}\right)\left\langle\overrightarrow{\mathrm{p}}_{1}, \epsilon_{2}|t| \overrightarrow{\mathrm{p}}_{1}, \epsilon_{1} ; \overrightarrow{\mathrm{k}}^{\prime} ;-\overrightarrow{\mathrm{k}}^{\prime}\right\rangle$,

where $G\left(k^{\prime}\right)$ describes the profile of the laser pulse. To simplify the calculation, the functions $G\left(k^{\prime}\right)$ and $g\left(\omega_{0}\right)$ are assumed to have Gaussian shapes,

$$
\begin{aligned}
& G\left(k^{\prime}\right)=\exp \left[-A\left(k^{\prime}-k\right)^{2}\right], \\
& g\left(\omega_{0}\right)=\exp \left[-B\left(2 \omega^{\prime}-\omega_{0}\right)^{2}\right],
\end{aligned}
$$

where

$$
\omega^{\prime}=k^{\prime} c .
$$

The constant $A$ is related to the laser pulse duration, the constant $B$ to the lifetime of the atomic state. After performing the integration in Eq. (7) and eliminating irrelevant factors, one has

$$
\begin{aligned}
\left\langle\overrightarrow{\mathrm{p}}_{1}, \epsilon_{2}|t| \overrightarrow{\mathrm{p}}_{1}, \epsilon_{1} ; \overrightarrow{\mathrm{k}} ;-\overrightarrow{\mathrm{k}}\right\rangle\left\{1+a \exp \left[i \psi-L^{2} /\left(2 A+4 c^{2} B\right)\right]\right\} \\
\quad \times \exp \left\{-\left[A B /\left(A+2 c^{2} B\right)\right]\left(2 \omega-\omega_{0}\right)^{2}\right\},
\end{aligned}
$$

where

$$
\psi=2 L\left[k-c B\left(2 \omega-\omega_{0}\right) /\left(A+2 c^{2} B\right)\right] .
$$

In reaching Eq. (11) it is assumed that the reduced transition amplitude is a slowly varying function of the wave vector $\overrightarrow{\mathrm{k}}$. In the experiment ${ }^{7}$ the laserpulse duration $\left(\sim 5 \times 10^{-9} \mathrm{sec}\right)$ is much shorter than the lifetime of the atomic state $\left(\sim 5 \times 10^{-8} \mathrm{sec}\right)$; this means that $A<c^{2} B$. From Eqs. (11) and (12) the resonance fringe variation can be expressed as

$$
\cos \left(L \omega_{0} / c\right) \exp \left(-L^{2} / 4 c^{2} B\right) .
$$

In reaching Eq. (13) the term $A$ has been dropped. Equation (13) indicates that the fringe variation only depends on the difference $L$, not the laser-beam frequency $\omega$, and that at large path-length difference the fringe contrast becomes very poor. The predicted fringe variation was actually observed experimentally. 8,9

It is sometimes claimed ${ }^{1,7}$ that to observe resonance fringes, the laser beams should be spatially or temporally separated. If in the performed experiment a continuous-wave laser were used, the resonance fringe variation would have the form $\left[1+a+2 a^{1 / 2} \cos (k L)\right]^{2}$. The continuous-wave laser has a very long.wave train, which can be longer than the path-length difference $L$. After the beam splitter, two overlaid beams are neither spatially nor temporally separated. Hence the requirement of beam separation is unnecessary.

The only relevant factor is the introduction of a direct phase into the coherent transition amplitude. In the experiment proposed by Baklanov, Dubetskii, and Chebotayev, ${ }^{3}$ the atomic transitions occur at completely different times. The transition amplitude can be written as

$$
\begin{aligned}
& \left\langle\overrightarrow{\mathrm{p}}_{1}, \epsilon_{2}|t| \overrightarrow{\mathrm{p}}_{1}, \epsilon_{1} ; \overrightarrow{\mathrm{k}} ;-\overrightarrow{\mathrm{k}}\right\rangle \\
& +\left\langle\overrightarrow{\mathrm{p}}_{1}, \epsilon_{2}\left|e^{i T H / \hbar} t e^{-i T H / \hbar}\right| \overrightarrow{\mathrm{p}}_{1}, \epsilon_{1} ; \overrightarrow{\mathrm{k}} ;-\overrightarrow{\mathrm{k}}\right\rangle \\
& =\left\langle\overrightarrow{\mathrm{p}}_{1}, \epsilon_{2}|t| \overrightarrow{\mathrm{p}}_{1}, \epsilon_{1} ; \overrightarrow{\mathrm{k}} ;-\overrightarrow{\mathrm{k}}\right\rangle \\
& +\left\langle\overrightarrow{\mathrm{p}}_{1}, \epsilon_{2}|t| \overrightarrow{\mathrm{p}}_{1}, \epsilon_{1} ; \overrightarrow{\mathrm{k}} ;-\overrightarrow{\mathrm{k}}\right\rangle e^{i\left(\omega_{0}-2 \omega\right) T},
\end{aligned}
$$

where $H$ is the total Hamiltonian of the system and $T$ the time of flight. Its fringe variation was given in Eq. (1). To achieve coherence in the atom transitions, the time of flight $T$ must be less than the lifetime $\tau$ of the atomic state $(T<\tau)$. At the present, the above condition cannot be satisfied experimentally. ${ }^{7}$

In the crossed-beam experiment, the direct phase is introduced through the laser beams. The laser beams in Fig. 1, which are responsible for the Doppler-free two-photon transition, can be expressed as $^{10}$

$$
\left|\overrightarrow{\mathrm{k}}_{1} ;-\overrightarrow{\mathrm{k}}_{1}\right\rangle+a e^{2 i k L}\left|\overrightarrow{\mathrm{k}}_{2} ;-\overrightarrow{\mathrm{k}}_{2}\right\rangle \text {. }
$$

The associated transition amplitude has been given in Eq. (2). The incident beam $\vec{k}_{2}$ and the reflected beam $-\overrightarrow{\mathrm{k}}_{2}$ each contribute a phase shift $k L$, which in turn leads a total shift $2 k L$ in Eq. (15). In the case of the pulsed laser, the above phase shift can possibly be interpreted as due to a temporal separation. In the crossed-beam experiment with pulsed lasers, to observe interference fringes the following equation has to be satisfied:

$$
(L / c)^{2} \leq\left(\tau_{e}^{2}+2 \tau^{2}\right) / 2,
$$

where $\tau_{e}$ is the duration of the pulse. It is obtained directly from Eq. (11).

The resonance fringe variations in Eqs. (1), (5), and (13) cannot be blurred by the laser beam momentum uncertainty associated with the finite size of the beam cross section. The transit time of atoms is determined by the cross-sectional width of the laser beam. This means that, even in the presence of the transit time broadening, the resonance fringes still can be observed. The observation of these fringes will lead to the improvement of spectral resolution. ${ }^{4}$

The underlying mechanics of coherence in the separated-beam and crossed-beam experiments are different. In the separated-beam experiment, the direct phase is introduced into the coherent transition amplitude through the displacement of the whole quantum system. Whether the laser beam is continuous or pulsed, for a fixed $T$, the resonance fringe variation with respect to the laser-beam fre- 
quency, as expressed in Eq. (1), is centered at exactly half the Bohr frequency. In the crossed-beam experiment, the direct phase is introduced into the coherent transition amplitude through the displacement of a part of the quantum system. It is an asymmetrical shift. Whether the laser beam is continuous or pulsed, for a fixed $L$, the resonance fringe variation with respect to the laser-beam frequency, as expressed in Eq. (11), is not centered at half the Bohr frequency. The resonance fringe variation in the crossed-beam experiment depends on variables $\omega=k c$ and $L$. It is always possible to vary these two variables together for the purpose of creating a particular fringe variation: for example, by setting ${ }^{11}$

$$
L \omega_{0} / c=2 n \pi-10 L \omega / c,
$$

where $n$ is an integer. Then the resonance fringe variation in Eq. (13) with respect to the laser beam frequency has a fringe spacing $3 c / 5 L$.

The observed phenomenon on the resonance fringes does not depend on detailed dynamics. In their theoretical discussion, Baklanov, Chebotayev, and Dubetskii ${ }^{3}$ used two-level dynamics in the weakfield approximation. In the discussions of Eqs. (14), (15), and (1), no detailed dynamics were employed. The observed phenomenon follows directly from basic quantum mechanics.

The resonance fringe variation in Eq. (13) is for an absorption line without a further hyperfine split- ting. But with a splitting $\Delta \omega_{0}$, a beating phenomenon occurs and the resonance fringe variation has the form

$$
\cos \left(L \omega_{0} / c\right) \cos \left(L \Delta \omega_{0} / 2 c\right) \exp \left(-L^{2} / 4 c^{2} B\right) .
$$

The laser-pulse duration does not impose a limit on the allowable range of the path difference $L$. As shown in Eq. (11), one can increase the fringe contrast by setting $\omega=\frac{1}{2} \omega_{0}$. Hence through the study of the transition rate dependence on the difference $L$, one can resolve the hyperfine splitting $\Delta \omega_{0}$ even with a short pulsed laser. In a conventional onelaser beam experiment, the hyperfine splitting resolution is limited by the laser-pulse duration.

In an actual experiment, the transition amplitude $\left\langle\vec{p}_{1}, \epsilon_{2}|t| \vec{p}_{1}, \epsilon_{1} ; \vec{k} ;-\vec{k}\right\rangle$ depends on the polarizations of the atomic and laser beams. This dependence leads to a polarization spectroscopy. In the crossed-beam experiment, besides the polarization dependence, the transition amplitude in Eq. (2) also depends on the relative strength $a$, path difference $L$, and intersection angle of the two coherent beams. One can label an absorption line according to these variables: for example, at what values of these variables the atomic absorption of a particular line is minimum. This is a new kind of spectroscopy.

The author is very grateful to Professor C. N. Yang, Professor V. L. Teplitz, and Professor J. A. Jacobs for numerous interesting discussions.
${ }^{1}$ See, for example, T. W. Hänsch, Phys. Today 30 (No. 5), 34 (1977).

${ }^{2}$ Ming Chiang Li, Nuovo Cimento 39B, 165 (1977).

${ }^{3}$ Ye. V. Baklanov, B. Ya. Dubetskii, and V. P. Chebotayev, Appl. Phys. 9 , 171 (1976); Ye. V. Baklanov, B. Ya. Dubetskii, and V. P. Chebotayev, Appl. Phys. 11, 120 (1976).

${ }^{4}$ N. F. Ramsey, Molecular Beams (Oxford Uniyersity, New York, 1956).

${ }^{5} \mathrm{~A}$. Gold, in Quantum Optics, Proceedings of the International School of Physics, Enrico Fermi (Course $X L I I$ ), edited by R. J. Glauber (A'cademic, New York, 1969)..

${ }^{6}$ The direct-phase determination is of interest by itself. Recently, there has been considerable experimental activity on the direct phase measurement of quantummechanical excitation amplitudes. See, for example,
H. Kleinpoppen, Comments Atom. Mol. Phys. $\underline{6}$ (2), 34 (1976); M. Standage and H. Kleinpoppen, Phys. Rev. Lett. 36,577 (1976).

${ }^{7}$ M. M. Salour and C. Cohen-Tannoudji, Phys. Rev. Lett. $\underline{38}, 757$ (1977).

${ }^{8}$ M. M. Salour, Bull. Am. Phys. Soc. 21, 1245. (1976).

${ }^{9}$ In Refs. 7 and 8 , these authors referred to the quantity $T_{e}=L / c$ as the time delay between two pulses. In terms of $T_{e}$, Eq. (13) can be rewritten as $\cos \left(\omega_{0} T_{e}\right) \exp \left(-T_{e}^{2} / 4 B\right)$. The corresponding fringe variation in these references is rewritten as $\cos \left(\omega_{0} T_{e} / 2\right)$. The factor of $\frac{1}{2}$ is an error.

${ }^{10}$ In the case of two overlaid continuous-wave laser beams $\left(\vec{k}_{1}=\vec{k}_{2}=\vec{k}\right)$, Eq. (15) should be replaced by $\left[1+a^{1 / 2} \exp (i k L)\right]^{2}|\overrightarrow{\mathrm{k}} ;-\overrightarrow{\mathrm{k}}\rangle$.

${ }^{11}$ In Ref. 7 , the experimenters set $L \omega / c=2 n \pi$, which in turn yields a fringe spacing $c / 2 L$. 